\section{Single Scattering of Fast $\beta$-Particles by Protons}

WE have recently taken some eight hundred pairs of photographs corresponding to about 124 metres of track of $\beta$-particles, of energies from 0.5 to $1.1 \mathrm{mV}$., in a mixture of 80 per cent hydrogen and 20 per cent oxygen, contained in an expansion chamber. Assuming that the scattering of the $\beta$-particles in oxygen is similar to that of the adjacent element nitrogen, for which results have already been obtained ${ }^{1}$, the elastic scattering of these $\beta$-particles through angles greater than $20^{\circ}$ by protons is found to be in agreement with Mott's theory. The theory predicted that about one such deflexion was to be expected ; actually two cases were observed. The statistical fluctuations were therefore large, but it may be inferred that there is no serious discrepancy between existing theory and experiment for the collisions of protons and electrons of this energy.

As in the previous work with nitrogen, no examples of inelastic collision of electrons and protons were observed.

King's College, London, W.C.2.

R. R. RAY

F. C. Champion Oct. 30.

${ }^{1}$ Champion, F. C., Proc. Roy. Soc., A, 153, 383 (1936).

\section{X-Ray Study of Noble Metals Dispersed in Borax- and $\mathrm{B}_{2} \mathrm{O}_{3}$-Glasses}

Is a previous communication ${ }^{1}$, it was shown by us that alkali halides such as lithium, sodium, potassium, rubidium and cæsium chloride dissolved in fused boric oxide giving a homogeneous glass. X-ray examination of these glasses reveals sharp diffraction lines due to the dissolved alkali halides together with a few foreign lines which cannot be identified with any known borates of the metals. These foreign lines are largely present in glasses containing chlorides of alkali metals of low atomic numbers, but are absent with rubidium and cæsium halides. It was found later that these foreign lines are due to a variety of anhydrous crystalline $\mathrm{B}_{2} \mathrm{O}_{3}$, first studied by Cole and Taylor ${ }^{2}$, in the case of boric oxide glasses, and to anhydrous $\mathrm{Na}_{2} \mathrm{~B}_{4} \mathrm{O}_{7}$ in the case of borax glasses.

The manner in which the alkali halide lattice exists within the non-repeating meshwork of boric oxide and borax glasses requires elucidation. Accepting Warren and Zachariasen's views about the structure of these glasses, one would be led to suggest that a new lattice is formed within the hollows of the cages formed by the $\mathrm{B}-\mathrm{O}-\mathrm{B}$ triangular bondage $\mathrm{But}$ in such a case the electrostatic forces between the ions should be diminished, as also probably the shortdistance repulsive forces, on account of the shielding action produced by a medium of higher dielectric constant necessitating an enlargement of the lattice. Preliminary experiments by Majumdar and Palit ${ }^{3}$ seemed to point to such a conclusion, but later work has shown that the spacings are almost unaltered in the glass.

An interesting case has been found with gold and platinum dispersed in boric oxide and borax glasses. The samples were prepared by first evaporating solutions of auric and platinic chlorides separately in a platinum crucible and then igniting, whereby the noble metals were obtained in a finely divided state. The reduced metals were then heated with specially

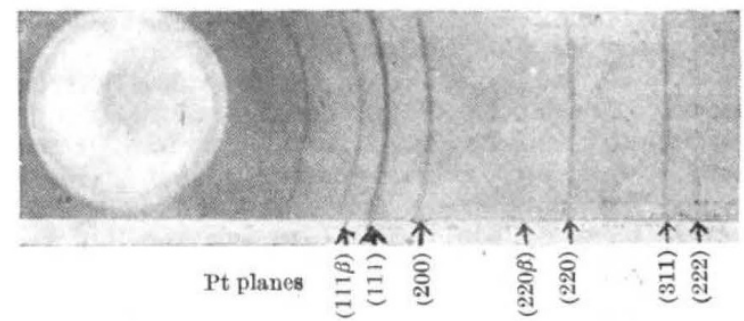

purified anhydrous $\mathrm{B}_{2} \mathrm{O}_{3}$ and $\mathrm{Na}_{2} \mathrm{~B}_{4} \mathrm{O}_{7}$ respectively at $800^{\circ}-1,000^{\circ}$ C., until thoroughly homogeneous (slightly coloured) masses were obtained. The crucible was then chilled and the solid extracted. Each piece of glass was examined under the polarization microscope for optical isotropy. The glass was then powdered, inserted in a capillary tube, and a DebyeScherrer photograph taken with a circular camera (radius $3.90 \mathrm{~cm}$.) and a Hadding tube with copper anti-cathode. The exposure varied from 8 to 10 . hours. Photographs were also taken of pure gold and platinum wires and the prints compared. A typical print of platinum dissolved, in $\mathrm{B}_{2} \mathrm{O}_{3}$-glass is reproduced. The following table gives the distance $x$ in cm. between two similar lines:

$\begin{array}{lccccccccc}x & 3 \cdot 35 & 3 \cdot 65 & 4 \cdot 0 & 4 \cdot 70 & 5 \cdot 225 & 5 \cdot 50 & 6 \cdot 10 & 7 \cdot 95 & 8 \cdot 95 \\ \text { Nature } & \mathrm{w} & \mathrm{s} & \mathrm{r} . \mathrm{w} & \mathrm{m} & \mathrm{s} & \mathrm{w} & \mathrm{s} & \mathrm{w} & \mathrm{s} \\ x \ldots & & 9 \cdot 55 & 10 \cdot 80 & 11 \cdot 40 & 15 \cdot 80 & 16 \cdot 50 & \\ \text { Nature } & & \mathrm{w} & \mathrm{s} & \mathrm{w} & \mathrm{s} & \mathrm{s} & \end{array}$

$$
\mathrm{w}=\text { weak, } \mathrm{r} . \mathrm{w}=\text { rather weak, } \mathrm{s}=\text { strong, } \mathrm{m}=\text { moderate. }
$$

By trial and error method, the constant for $K \propto(1.539 \mathrm{~A}$.$) is found to be 0 \cdot 1954$, and for $K \beta$ (1.389 A.) 0.1756 . Hence the spacing $a_{0}$ for platinum dissolved in glass works out as follows :

$$
\begin{aligned}
& \text { for } K \alpha, a_{0}=\frac{1.539 \times 10^{-8}}{2 \times 0.1954}=3.938 \mathrm{~A} \text {, } \\
& \text { and for } K \beta, a_{0}=\frac{1.389 \times 10^{-8}}{2 \times 0.1756}=3.950 \mathrm{~A} \text {., }
\end{aligned}
$$

the mean value, $3.944 \mathrm{~A}$., being in good agreement with the standard value for platinum, namely, $3 \cdot 910 \mathrm{~A}$.

$$
\text { Presidency College, }
$$

Subodh Kumar MaJumdar Bhupati Kumar BanerJee and

Indian Association for the

Cultivation of Science, Calcutta.

${ }^{1}$ Nature, 156, 423 (1945).

${ }^{2}$ J. Amer. Chem. Soc., 56, 1648 (1934).

${ }^{3} J$. Indian' Chem. Soc., 19, 461 (1942).

\section{Cerium Tungstate as a Semi-Conductor}

Вотн Zambonini ${ }^{1}$ and Beintema ${ }^{2}$ claim to have prepared tetragonal bipyramidal crystals of cerium tungstate, $\mathrm{Ce}_{2}\left(\mathrm{WO}_{4}\right)_{3}$, by fusing a precipitate, using sodium chloride as a flux. Subsequent work by Sillen and Sundvall ${ }^{3}$ showed that the compound was actually $\mathrm{NaCe}\left(\mathrm{WO}_{4}\right)_{2}$ of scheelite-type structure. Tammann ${ }^{4}$ has also described a cerium tungstate, but the only observations made were on the change with tempera. ture of electrical resistance of powdered compacts. The resistance of a pellet $1 \mathrm{~mm}$. thick and $14 \mathrm{~mm}$. diameter was given as $40,000 \Omega$ at $600^{\circ} \mathrm{C}$. and $1,700 \Omega$ at $800^{\circ} \mathrm{C}$.

In an investigation which required the use of fired mixtures of cerium oxide $\left(\mathrm{CeO}_{2}\right)$ and tungstic oxide 